Hydride Precipitation Kinetics in Zircaloy-4 Studied Using Synchrotron X-Ray Diffraction

Authors: Olivier F. Courty, Arthur T. Motta, Christopher J. Piotrowski, Jonathan D. Almer

\title{
Olivier Courty
}

Pennsylvania State University

45 Bd Gouvion Saint Cyr

75017 Paris

FRANCE

Phone: +33677104525

Fax : +33170756303

o.courty@gmail.com

Arthur T. Motta (corresponding author)

Pennsylvania State University

Department of Mechanical and Nuclear Engineering

227 Reber Building

Penn State University, University Park, PA, 16802

United States

phone: $\underline{814-865-0036}$ fax: $\underline{814-865-1280}$

atm2@.psu.edu

\section{Christopher J. Piotrowski}

Pennsylvania State University

Department of Mechanical and Nuclear Engineering

208 Hammond Building

Penn State University, University Park, PA, 16802

United States

cjp5169@psu.edu

Jonathan D. Almer

Argonne National Laboratory

9700 S. Cass Ave

431/A006

Argonne, IL 60439

United States

Phone: 630-252-1049 Fax: 630-252-5391

almer@aps.anl.gov 


\section{Introduction}

The zirconium alloy nuclear fuel cladding in the core of a Light Water Reactor (LWR) undergoes corrosion and hydrogen pickup by the primary circuit water [1,2], which can result in the precipitation of the hydrogen into brittle hydride particles and which may reduce cladding ductility. The hydrogen which enters the cladding can exist in two different states: in solid solution within the zirconium matrix and precipitated as zirconium hydrides. The hydrogen in solid solution corresponds to hydrogen atoms occupying interstitial sites in the hcp zirconium structure. Once the concentration of hydrogen in solid solution exceeds the Terminal Solid Solubility limit for precipitation (TSSp), hydrogen atoms agglomerate into hydride platelets [3].

The kinetics of hydrogen precipitation is a critical aspect of hydrogen redistribution in the cladding. While the TSSp and TSSd (Terminal Solid Solubility for dissolution) determine the equilibrium value between the hydrogen in solid solution and in the precipitated hydrides, the kinetics provides information on the approach to this equilibrium.

Hydride precipitation from supersaturated solid solution has been described in the model developed by Marino [4, 5], in which the precipitation rate is taken to be proportional to the amount of hydrogen supersaturation in solid solution. The supersaturation is given by the difference between the hydrogen concentration in solid solution $\mathrm{C}_{\mathrm{ss}}$, and the equilibrium value, given by the TSSp. The rate of hydrogen atoms leaving solid solution to be absorbed into hydrides is:

$$
\frac{d C_{s s}}{d t}=-\alpha^{2}\left(C_{s s}-T S S p\right)
$$

Marino measured the kinetic parameter $\alpha^{2}$ to be $2 \times 10^{-4} \mathrm{~s}^{-1}$ at $300^{\circ} \mathrm{C}$. The same parameter $\alpha^{2}$ was also measured by Kammenzind, who suggested an Arrhenius law to describe its dependence on temperature [6]. His experiment is based on simultaneous diffusion and precipitation. Therefore the calculation of the kinetics parameter depends on the value chosen for the diffusion coefficient. The equation found for the kinetics parameter is:

$$
\alpha^{2}=A_{\alpha}^{2} \exp \left(\frac{2 Q_{\alpha}}{R T}\right)
$$

with $A_{\alpha} \sim 62.3 \mathrm{~s}^{-1 / 2}$ and $Q_{\alpha} \sim 41.2 \mathrm{~kJ} / \mathrm{mole}$.

Subsequent studies of the precipitation of hydrogen in zirconium were recently performed using neutron diffraction [7, 8] and X-ray diffraction [9]. Zanellato et al., measured the precipitation kinetics at $400^{\circ} \mathrm{C}$, using a similar technique as used in this paper, finding $\alpha^{2}=0.012$. No systematic measurement of at different temperatures was performed in that study [9].

The goal of this study is to validate the linear approximation of Marino's phenomenological law given by equation (1) and to measure directly the value of $\alpha^{2}$ at different temperatures using different initial concentrations. 


\section{Experimental Geometry and Material}

The material used for the experiments was cold-worked stress-relieved Zircaloy-4 sheet machined into coupons of dimensions $1 \mathrm{~cm} \times 4.2 \mathrm{~cm}$ and $660 \mu \mathrm{m}$ thick. The samples were recrystallized at $640^{\circ} \mathrm{C}$ for 7 minutes, and then cooled down under high vacuum $\left(<1 \times 10^{-5}\right.$ Torr). Once recrystallized, these samples were hydrogen-charged by high temperature gas diffusion, performed in several steps. First, the native oxide layer present on the surface of the samples was removed by dipping the samples for $1 \mathrm{~min}$ in an acid solution of one part HF, 10 parts nitric acid and 10 parts $\mathrm{H}_{2} \mathrm{O}$. Second, using an electron-beam evaporator a $100 \mathrm{~nm}$ layer of nickel was deposited on each of the sample surfaces to prevent air oxidation. The samples were then hydrogen-charged in a vacuum furnace using a mixture of $12 \%$ hydrogen and $88 \%$ argon, introduced at $500^{\circ} \mathrm{C}$. For each sample, small amounts of material were cut off for chemical analysis of hydrogen, performed by Luvak, Inc., using vacuum hot extraction. The measured hydrogen content of the samples is shown in Table 1. The TSSd measured during the experiment was another technique used for estimation of hydrogen content as was done in [10].

\section{Experimental Procedure and Results}

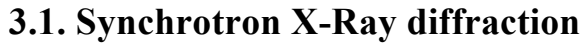

Samples were examined using high energy synchrotron radiation X-Ray diffraction at beamline 1-ID of the Advanced Photon Source (APS) at Argonne National Laboratory. As shown in Figure 1, the high X-ray energy used $(86 \mathrm{keV})$ allows the X-Ray beam to transmit through the sample thickness, thus providing bulk information on the sample studied. This beamline is equipped with an amorphous silicon Ge detector that allows fast data collection rates thus enabling the study of the time-dependent process of hydride precipitation. The incident x-ray beam is focused to a rectangular shape of $200 \times 200 \mu \mathrm{m}$ [11], which means that a volume of $10^{7} \mathrm{~m}^{3}$ (and thousands of $\mathrm{Zr}$ grains) is examined. In the beamline, an optical furnace can heat the samples up to $900^{\circ} \mathrm{C}$ while recording X-Ray diffraction data. The heating and cooling rates are monitored using K-type thermocouples, screwed to the sample surface and which provide feedback to the furnace. The sample is held by the grips and the diffraction patterns are recorded on the area detector behind. The geometry shown in Fig.1 allows the full diffraction rings to be recorded. These diffraction patterns contain both hydride and hep Zr matrix peaks.

The results of the fit of the X-ray diffraction data are used to measure the volume fraction of hydrides in the zirconium matrix. As mentioned above, the data is acquired on a large two-dimensional plate detector that allows the recording of the full diffraction rings in a wide range of two-theta angles. The detector recording area is $2048 \times 2048$ pixels with each pixel measuring $200 \times 200 \mu \mathrm{m}$, which gives an angular resolution of $4.6 \times 10^{-3}$ degrees (for $2 \theta$ ) for the typical set-up used. Continuous recording of data was performed to study the kinetics of hydride precipitation in situ. Each diffraction frame was recorded as the sum of ten consecutive images with a typical individual exposure time of $0.5-1$ second (this is done to avoid saturation of the detector). While recording data, the temperature was monitored and recorded using control computers. One full 10-exposure diffraction pattern series was typically taken every 10 seconds.

Several steps were performed to analyze the raw two-dimensional diffraction patterns. The ten images recorded for one frame were summed and averaged and the diffraction rings integrated over the whole azimuth using a Python APS subroutine and a Matlab ${ }^{\circledR}$ subroutine. The integration files obtained were then reduced to a one-dimensional intensity versus two-theta file for analysis using the GSAS program as 
illustrated in Figure 2. The fitted peak was the -hydride 111, which is convoluted with the hcp-Zr peak 100. The deconvolution of these two peaks was performed using GSAS. GSAS is a Rietveld analysis program which also allows to fit only the peak shape, position and intensity of the raw data using the Rawplot subroutine. The peaks are fitted using a pseudo-Voigt function which is a convolution of Gaussian and Lorentzian peak shapes. The background was modeled using a third-degree polynomial function and several refinement steps were iterated (usually 10 successive refinements for each parameter we chose to refine). This allowed us to fit the diffraction peaks and obtain the integrated intensity and the peak positions for the desired peaks.

\subsection{Thermal cycles}

Typical cladding temperatures in a PWR can range from $280^{\circ} \mathrm{C}$ to $400^{\circ} \mathrm{C}$. This study measures the precipitations kinetics at target temperatures of $288^{\circ} \mathrm{C}, 316^{\circ} \mathrm{C}, 332^{\circ} \mathrm{C}, 360^{\circ} \mathrm{C}, 380^{\circ} \mathrm{C}$ and $400^{\circ} \mathrm{C}$. To determine the kinetics parameter $\alpha^{2}$ a temperature cycle was used which consists of heating the sample to a temperature at which all the hydrogen is dissolved (i.e. $10^{\circ} \mathrm{C}$ above the dissolution temperature), then cooling quickly (about $1{ }^{\circ} \mathrm{C} / \mathrm{s}$ ) to the target temperature. This fast cooling produces a significant supersaturation of hydrogen in solid solution, as not all the hydrogen has time to precipitate. Once the temperature is stabilized at the target temperature, the sample is held at this temperature, while the supersaturated hydrogen precipitates. Diffraction patterns were acquired continuously (every 10s or so) and at high temperature no beta phase peaks were observed.

Figure 3 shows both the temperature profile and the delta-hydride 111 peak diffraction signal for the experiment performed on the sample containing $541 \mathrm{wt} . \mathrm{ppm}$. The kinetics of precipitation is followed by monitoring the hydride peak intensity. The figure illustrates the variation of the hydride peak intensity with time as the sample goes through the temperature cycle shown in the continuous black line. Initially at room temperature the hydride diffracted intensity is high as the TSSd at room temperature is low. As the temperature increases the hydride peak intensity decreases until it goes to zero at the dissolution temperature, when $\mathrm{TSSd}=\mathrm{C}_{\text {TOTAL }}$ (total hydrogen concentration), i.e. all the hydrogen is in solid solution. The top temperature of $545^{\circ} \mathrm{C}$ is then reached and from there the sample is cooled to various temperatures. In the first case the target temperature is $288^{\circ} \mathrm{C}$ then $312^{\circ} \mathrm{C}$, etc. Because TSSp (and consequently the hydride volume fraction) decreases with increasing temperature the intensity of the 111 delta hydride peak decreases proportionately.

In each case, however, the hydride peak diffraction intensity observed immediately upon cooling is lower than that obtained after further holding at the target temperature (the equilibrium value). The kinetics of this approach to equilibrium is the object of this study. The portions of the curve used for the determination of $\alpha^{2}$ are highlighted in green in Figure 3. This highlighted region corresponds the holding of the sample at a stable temperature as measured by the thermocouples and represents the crucial part of the experiment, when only hydride growth is expected to occur.

It is interesting to note that the hydrogen concentration of each sample can be confirmed by noting the dissolution temperature during the heatup phase and using the TSSd (terminal solid solubility for hydride dissolution) formula given by McMinn et al [12]. This was done for the samples in this study and the results were close to those of the determination by vacuum hot extraction, but somewhat different. Since the VHE results were obtained in sister samples and variations in hydrogen concentration can occur within a given sample, the hydrogen concentrations from the TSSd determination were judged to be the most accurate measure of hydrogen concentration and thus were used in this work. 


\subsection{Measurement of the kinetics parameter $\alpha^{2}$}

The precipitation kinetics is measured by following the approach to steady state of the hydride volume fraction while the temperature is held at a constant value. If Marino's model is correct, this approach should be well described by equation (1). Since the loss of hydrogen from solid solution corresponds to hydrogen precipitation at the hydrides, the change in the amount of hydrogen in the hydrides $\left(C_{p p}\right)$ and the amount in solid solution $C_{s s}$ is given by:

$$
\left\{\begin{array}{l}
\frac{d C_{p p}}{d t}=\alpha^{2}\left(C_{S S}-T S S p\right) \\
\frac{d C_{s s}}{d t}=-\alpha^{2}\left(C_{S S}-T S S p\right)
\end{array}\right\}
$$

According to the model, for a given holding temperature both the kinetics parameter $\alpha^{2}$ and TSSp are constant. Rearranging equation (1) :

$$
C_{s s}(t)=\left(C_{i n i}-T S S p\right) \exp \left(-\alpha^{2} t\right)+T S S p
$$

where $C_{i n i}$ is the overall concentration of hydrogen in solid solution when the target temperatures has just been reached, corresponding to the beginning of the kinetics analysis. The concentration of hydrogen in the hydrides is:

$$
C_{p p}(t)=-\left(C_{i n i}-T S S p\right) \exp \left(-\alpha^{2} t\right)+\text { constant }
$$

By conservation of mass,

$$
C_{\text {TOTAL }}=C_{p p}(t)+C_{S S}(t)=C_{p p}^{e q}+T S S p
$$

With $C_{\text {TOтAL }}$ being the total amount of hydrogen in the sample and $C_{p p}^{e q}$ the equilibrium amount of hydrogen in the hydrides. Rearranging:

$$
-\ln \left(C_{p p}^{e q}-C_{p p}(t)\right)=\alpha^{2} t-\ln \left(\mathrm{C}_{\mathrm{ini}}-T S S p\right)+\text { constant }
$$

Assuming that the intensity of the (111) delta hydride peak is proportional to the volume fraction of hydrides ${ }^{1}$, which is also proportional to the amount of hydrogen in hydrides (in wt.ppm), equation (7) can be rewritten as a function of the hydride diffracted intensity $I_{p p}$ and the same value at equilibrium $I_{p p}^{e q}$ :

$$
-\ln \left(I_{p p}^{e q}-I_{p p}(t)\right)=\alpha^{2} t-\ln \left(C_{i n i}-T S S p\right)+\text { constant }
$$

${ }^{1}$ This assumes that there is no variation in the preferential orientation of the hydrides. Although, this may be a strong assumption, it is a logical first step. 
By holding the samples at the target temperature for sufficient time, it can be assumed that by the end of the respective cycle the amount of hydrogen in the hydrides has reached the $C_{p p}^{e q}$. Following this assumption, the last $1 \%$ of the points in each case were averaged to determine the equilibrium value of the hydride peak intensity $I_{p p}^{e q}$ for the temperature of interest. Following equation (8), the natural log of the difference of the hydride diffracted intensity and the same value at equilibrium was plotted against holding time. If the process is linear, a straight line should be obtained and the slope of the line is then equal to $-\alpha^{2}$.

The three samples studied were taken to different series of target temperatures as shown in table 2 .

. Figure 4 and 5 show the experiments conducted for samples A and C, analogously to that for sample B shown in Figure 3

In the three cases studied the data was found to be well described by a linear relationship. One example of such a fit is shown in Fig.6. One should note that the determination of the equilibrium temperature was done from the peak intensity data by taking the last 1,2 or $5 \%$ of the points. Little difference was seen in the results when these different approaches were used, which supports the robustness of the approach. The fact that the kinetics are well described by a linear fit of the log plots such as in Fig. 6 supports the assumption that Marino's model is valid to describe the hydrogen precipitation kinetics.

\subsection{Results}

As shown in Table 2 the experiment was performed for six temperatures for the sample with 541 wt.ppm, three temperatures for the sample with 434 wt.ppm and three temperatures for the sample with 603 wt.ppm, using the approach illustrated in Figures 3-5, i.e., the sample was heated to a temperature above the hydride dissolution temperature and then cooled quickly to the various target temperatures. The approach to the equilibrium hydride volume fraction was then measured by following the delta hydride (111) peak intensity. Since the kinetics of precipitation follows Marino's model, the values of 2 can be determined by fitting. The values of 2 obtained using this method are summarized in Table 2. Figure 7 shows the value of $\alpha^{2}$ plotted against temperature for the values determined in this study. The values obtained are of the same order of magnitude as those obtained by Kammenzind [7] (although showing less temperature variation) but are significantly lower than the $\alpha^{2}=1.2 \times 10^{-2} \mathrm{~s}^{-1}$ value found by Zanelatto [9].

Figure 7 shows that the value of the precipitation rate 2 was consistent among samples and did not vary with different temperatures in the range studied. The one outlier is Sample C (603 wt ppm) measurement at $380^{\circ} \mathrm{C}$ for which a good fit was obtained with $\mathrm{a} 2=9.45 \times 10-4 \mathrm{~s}-1$, suggesting a moderate temperature dependence. The overall average value of $\alpha^{2}$ is $4.5 \times 10^{-4} \mathrm{~s}^{-1}$.

\section{Discussion}

The values measured for 2 are between $3.7 \times 10^{-4}$ and $5.2 \times 10^{-4} \mathrm{~s}^{-1}$ with little temperature dependence. These values are of the same order of magnitude as obtained in Kammenzind's measurements (from $10^{-4}$ to $10^{-3} \mathrm{~s}^{-1}$ ) [7]. The values found for 2 show a slight but consistent increase with temperature but it is considered that this increase is within experimental uncertainty. If this increase were significant, it would be 
in the direction of greater diffusion at higher temperature which causes faster kinetics, indicating that the hydrogen in solid solution would respond faster to the driving force.

The hydrogen precipitation kinetics measured in this experiment is likely determined by hydride growth rather than by hydride nucleation. It is indeed not possible to measure nucleation with this technique, since it happens during the fast cooling of the sample. Growth involves two distinct phenomena. First, the hydrogen has to diffuse toward a hydride particle. Second, a chemical reaction transforms the hydrogen and the $\mathrm{Zr}$ into a hydride (assumed $\delta$ hydride). One of these two phenomena should be the rate limiting step in the kinetics. If we consider that the kinetics is largely temperature independent a logical conclusion would be to attribute the rate limiting step to the hydrogen-hydride chemical reaction which causes the kinetic parameter to be constant, while noting that if the temperature dependence is real, some role should be ascribed to the diffusion of hydrogen.

\section{Conclusions}

An experiment has been designed and conducted to verify Marino's precipitation kinetics model and to determine the precipitation rate constant 2 at various holding temperature and hydrogen contents, using in situ x-ray diffraction. The conclusions are as follows:

1. The measurements of the precipitation kinetics of hydrogen in Zircaloy-4 are consistent with the linear approximation in the hydride precipitation phenomenological model proposed by Marino, i.e. the rate of precipitation was measured to be proportional to the hydrogen supersaturation.

2. The average precipitation rate coefficient 2 was determined to be $4.5 \times 10^{-4} \pm 0.4 \times 10^{-4} \mathrm{~s}^{-1}$. Only a slight dependence of temperature was measured for this coefficient, in contrast with previous work, and no dependence on the initial concentration of hydrogen was observed.

3. The in situ measurement of diffraction peaks using synchrotron X-Ray diffraction in transmission appears to be a suitable technique to determine hydride precipitation kinetics.

\section{Acknowledgements}

This research was funded by the U.S. Department of Energy Nuclear Energy University Programs (NEUP) project 11-2987. We thank Jun Park and the APS staff for their help in performing the experiments, and Mark Daymond for providing the Rawplot program and the Ceria files used in the analysis. We thank Donald Koss for helpful discussions and the Penn State Nanofab staff for their help in the preparation of the samples. Use of the Advanced Photon Source was supported by the U.S. Department of Energy, Office of Basic Energy Sciences under Contract No. DE-AC02-06CH11357.

\section{References}


[2] C. E. Coleman and D. Hardie, "The hydrogen embrittlement of $\alpha$ zirconium review," Journal of the Less Common Metals, vol. 11, pp. 168-185, 1966.

[3] Kearns, "Terminal Solubility and partitioning of hydrogen in the $\alpha$ phase of Zirconium, Zircaloy-2 and Zircaloy-4," Journal of nuclear Materials, vol. 22, pp. 292-303, 1967.

[4] G. P. Marino, "Hydrogen Supercharging in Zircaloy," Materials Science and Engineering, vol. 7, pp. 335-341, 1971.

[5] G. P. Marino, "HYDIZ: A 2-dimensonal computer program for migration of interstitial solutes of finite solubility in a thermal gradient (LWBR Development Program)," WAPD-TM-1157, 1974.

[6] B. F. Kammenzind, D. G. Franklin, H. R. Peters, and W. J. Duffin, "Hydrogen Pickup and Redistribution in $\alpha$-Annealed Zircaloy-4," Zirconium in the Nuclear Industry: 11th International Symposium, vol. ASTM STP 1295, pp. 338-370, 1996.

[7] W. M. Small, J. H. Root, and D. Khatamian, "Observation of kinetics of [gamma] zirconium hydride formation in Zr-2.5Nb by neutron diffraction," Journal of Nuclear Materials, vol. 256, pp. 102-107, 1998/8 1998.

[8] J. H. Root, W. M. Small, D. Khatamian, and O. T. Woo, "Kinetics of the [delta] to [gamma] zirconium hydride transformation in Zr-2.5Nb," Acta Materialia, vol. 51, pp. 2041-2053, 2003/4/18 2003.

[9] O. Zanellato, M. Preuss, J.-Y. Buffiere, F. Ribeiro, A. Steuwer, J. Desquines, et al., "Syncrhotron diffraction study of dissolution and precipitation kinetics of hydrides in Zircaloy-4," Journal of Nuclear Materials, vol. 420, pp. 537-547, 2012.

[10] K. B. Colas, A. T. Motta, J. D. Almer, M. R. Daymond, M. Kerr, A. D. Banchik, et al., "In situ study of hydride precipitation kinetics and re-orientation in Zircaloy using synchrotron radiation," Acta Materialia, vol. 58, pp. 6575-6583, 2010.

[11] D. R. Haeffner, J. D. Almer, and U. Lienert, "The use of high energy X-rays from the Advanced Photon Source to study stresses in materials," Materials Science and Engineering: $A$, vol. 399, pp. 120-127, 2005.

[12] A. McMinn, E. C. Darby, and J. S. Schofield, "The terminal solid solubility of hydrogen in zirconium alloys," in 12th Int. Symp. on Zr in the Nuclear Industry, Toronto, CA, 2000, pp. 173-195.

[13] A. McMinn, Darby, E.C., Schofield, J.S., "The Terminal Solid Solubility of Hydrogen in Zirconium Alloys," Zirconium in the Nuclear Industry: Twelfth International Symposium, vol. ASTM STP 1354, pp. 173-195, 2000. 
Table 1: Overall hydrogen concentration in the APS samples measured by two different techniques

\begin{tabular}{|c|c|c|}
\hline Sample Designation & Content (wt.ppm) & $\begin{array}{c}\text { Measurement using } \\
\text { TSSd (wt ppm) }\end{array}$ \\
\hline A & $400 \pm 30$ & $434 \pm 40$ \\
\hline B & $541 \pm 24$ & $616 \pm 60$ \\
\hline C & $603 \pm 41$ & $682 \pm 70$ \\
\hline
\end{tabular}


Table 2: Measurement of the kinetics parameter $\alpha^{2}$

\begin{tabular}{|c|c|c|c|}
\hline \multirow{4}{*}{$\mathrm{A}$} & $\mathrm{T}\left({ }^{\circ} \mathrm{C}\right)$ & $\alpha^{2} \times 10^{-4}\left(\mathrm{~s}^{-1}\right)$ & $\mathrm{R}^{2}(\%)$ \\
\hline \multirow{4}{*}{$\mathrm{N}$} & 288 & 3.76 & 63.5 \\
\cline { 2 - 4 } & 316 & 5.25 & 88.1 \\
\hline \multirow{4}{*}{$\mathrm{B}$} & 332 & 4.91 & 93.1 \\
\cline { 2 - 4 } & 288 & 3.76 & 64.9 \\
\cline { 2 - 4 } & 316 & 3.62 & 78.8 \\
\cline { 2 - 4 } & 332 & 4.83 & 84.3 \\
\cline { 2 - 4 } & 360 & 4.34 & 88.9 \\
\cline { 2 - 4 } & 380 & 4.70 & 92.5 \\
\hline \multirow{4}{*}{$\mathrm{C}$} & 400 & 4.97 & 91.9 \\
\cline { 2 - 4 } & 316 & 4.55 & 77.9 \\
\cline { 2 - 4 } & 332 & 4.23 & 87.7 \\
\cline { 2 - 4 } & 360 & 5.29 & 88.7 \\
\cline { 2 - 4 } & 380 & 9.45 & 92.1 \\
\hline
\end{tabular}




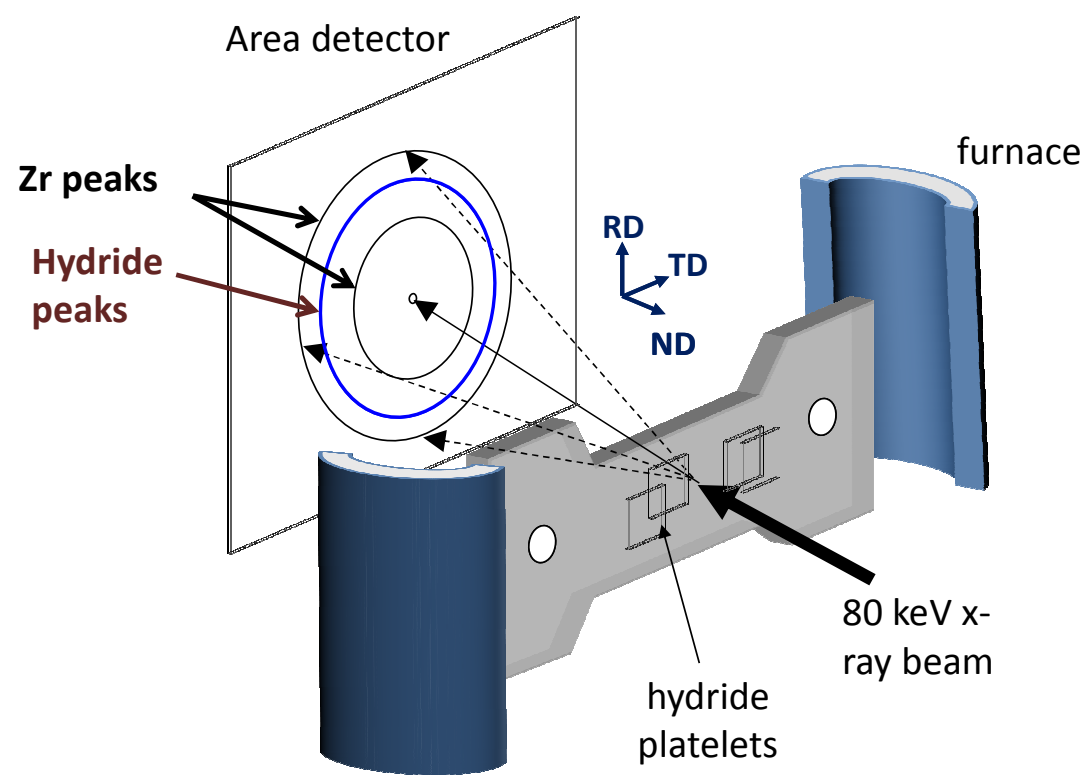

Figure 1: Schematic of the high energy $X$-ray beam and the area detector to record diffraction patterns as a function of sample temperature 


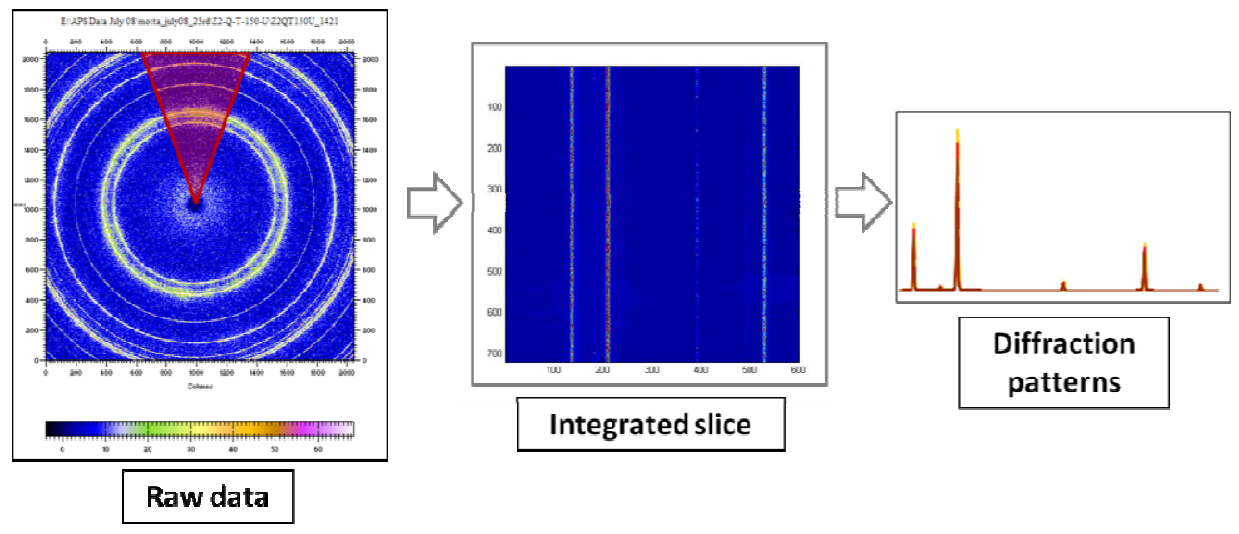

Figure 2: Schematic of the integration of the X-Ray diffraction data collected at beamline 1-ID. A section of the diffraction circle or the whole circumference can be integrated, as desired. 


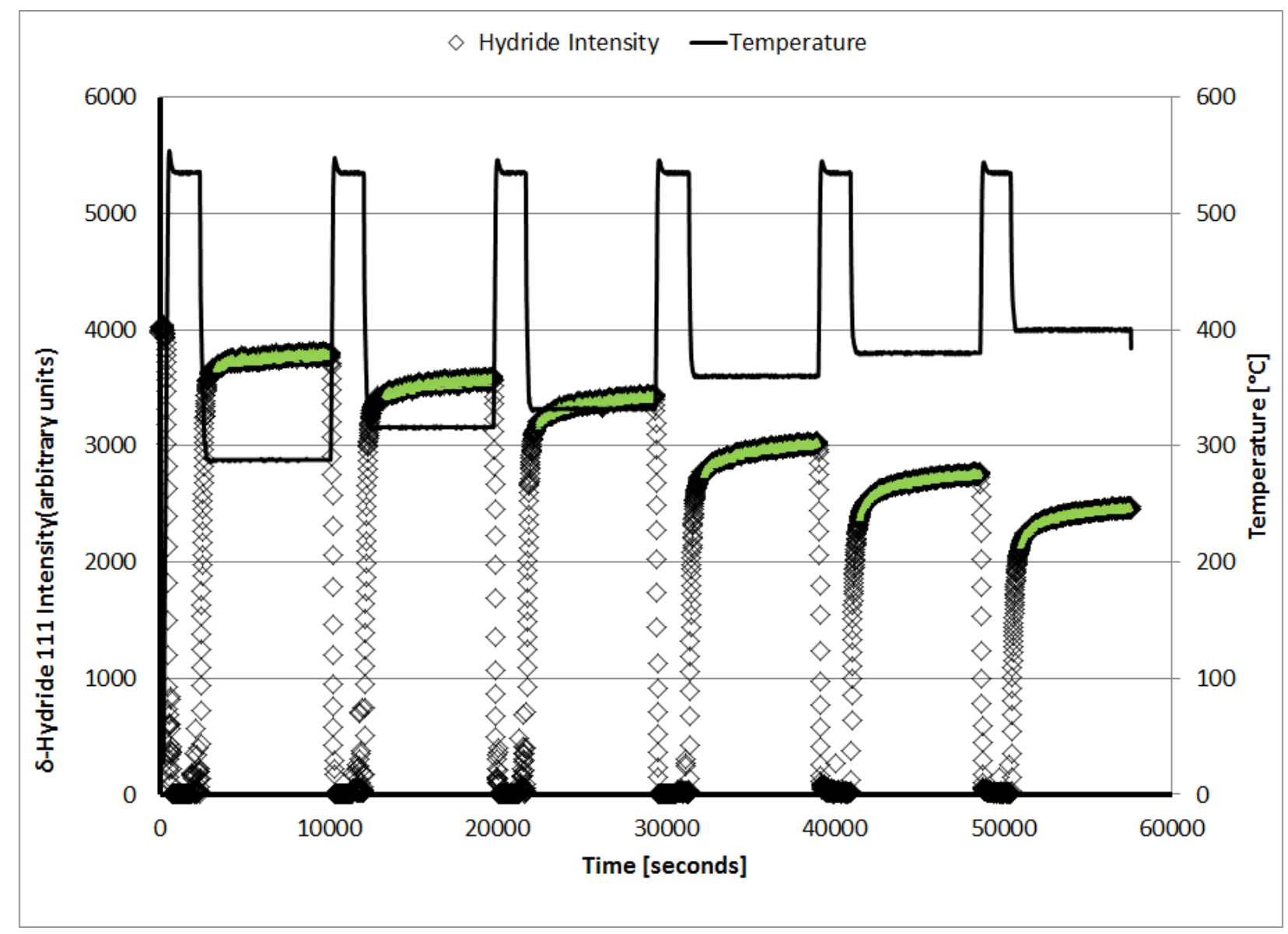

Figure 3: Temperature and hydride 111 diffraction signal for the experiments performed on sample B (containing 541 wt.ppm H). 


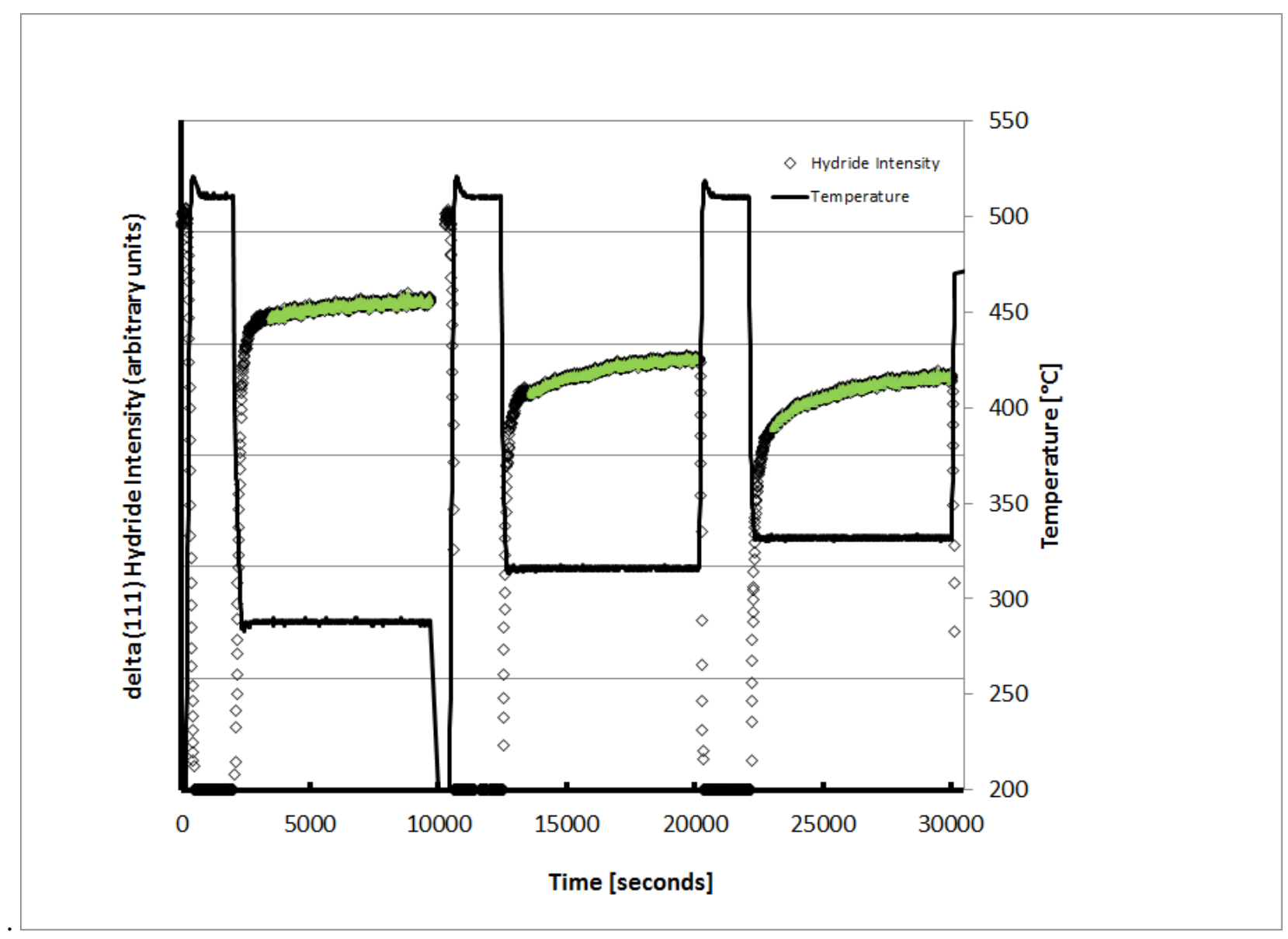

Figure 4: Temperature and delta hydride 111 diffraction intensity for the experiments performed on sample A (containing 400 wt.ppm H). 


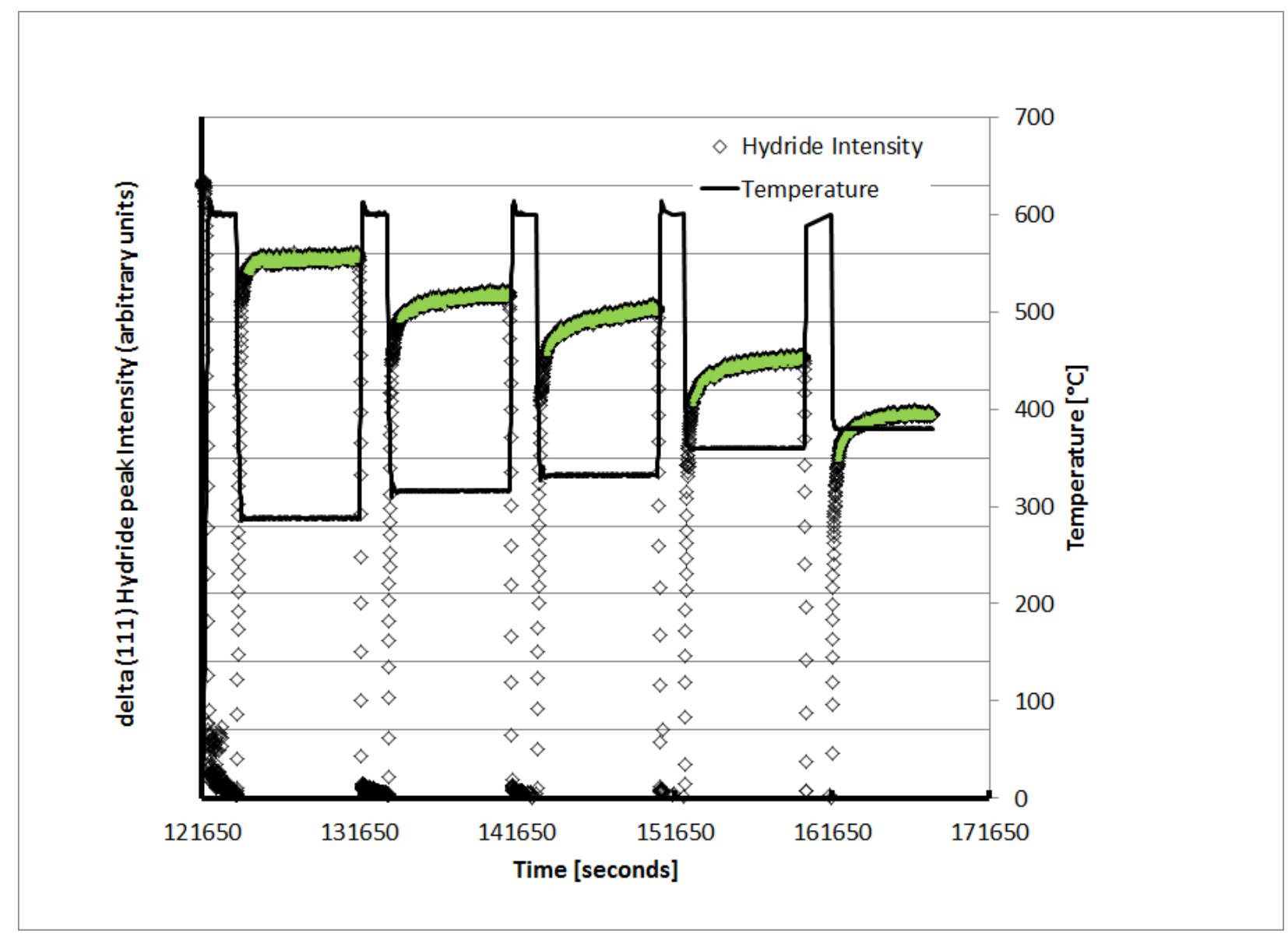

Figure 5: Temperature and hydride 111 diffraction signal for the experiments performed on sample C (containing 603 wt.ppm H). 


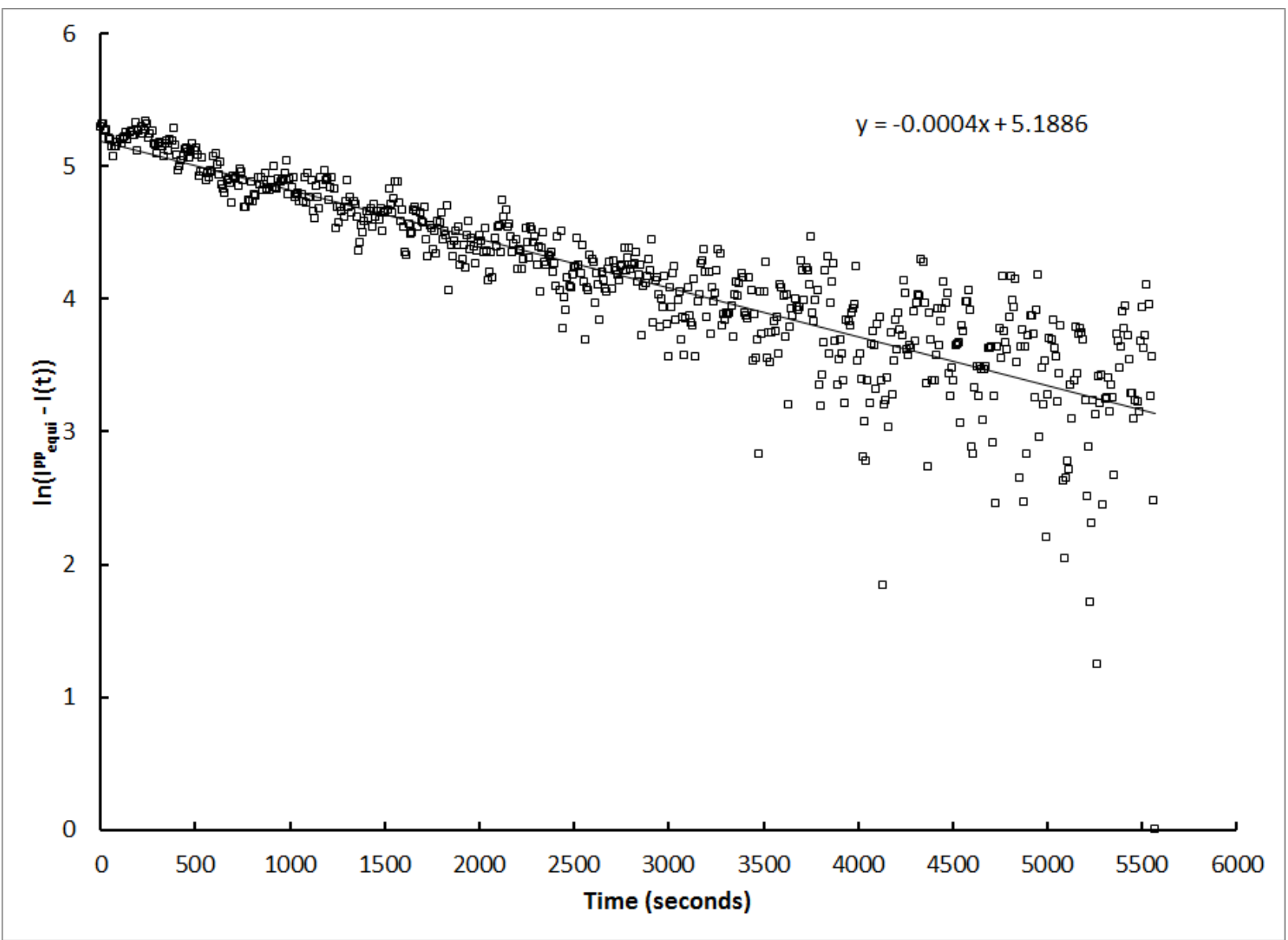

Figure 6: Hydrogen kinetics and determination of $\alpha^{2}$ for sample B (541 wt.ppm) when the target temperature for holding was $316^{\circ} \mathrm{C}$. The slope in this case was $-\alpha^{2}=3.62 \times 10^{-4} \mathrm{~s}^{-1}$. 


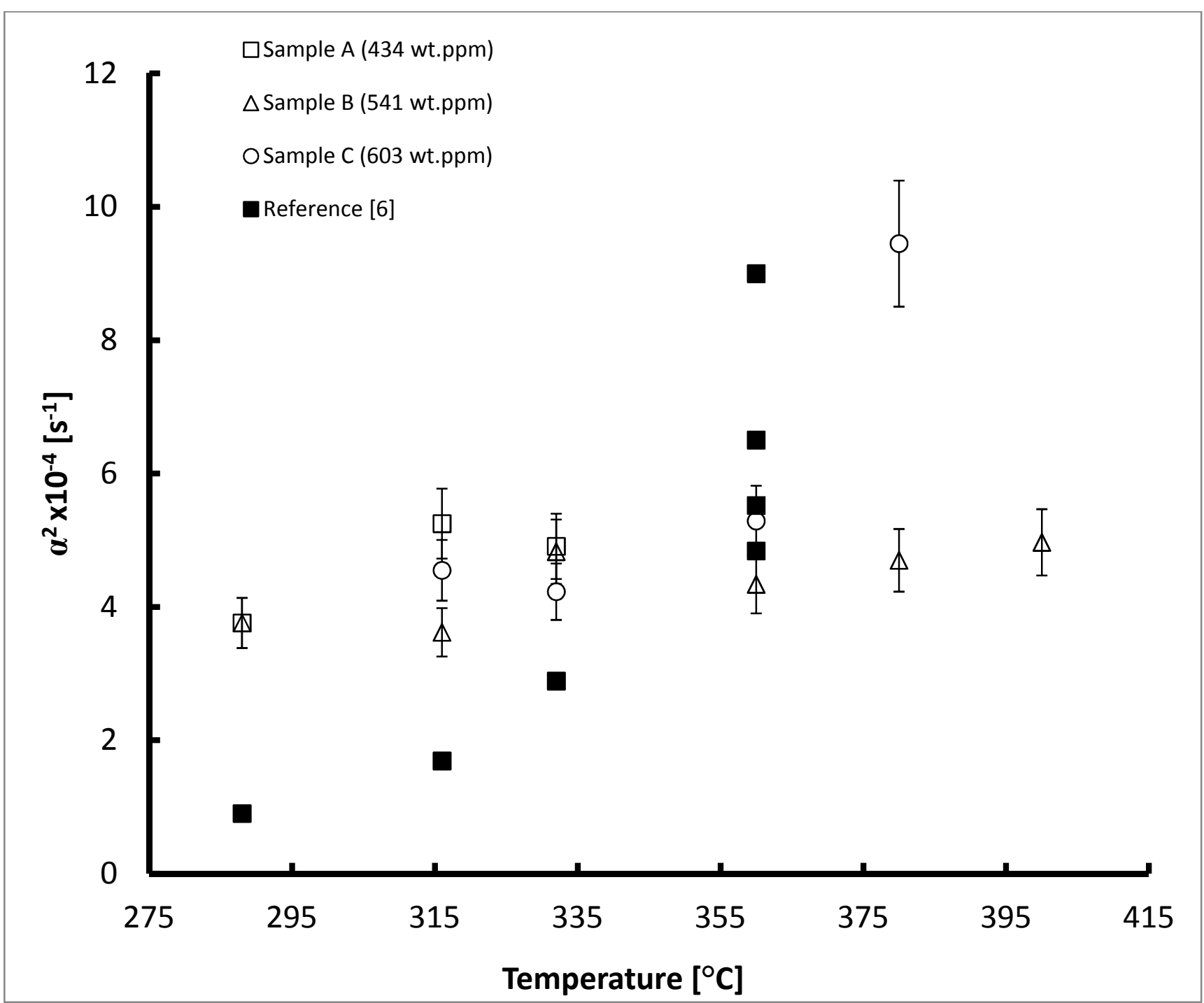

Figure 7: Kinetic parameter $\alpha^{2}$ versus holding temperature, with different initial concentrations compared to ref [6]. 